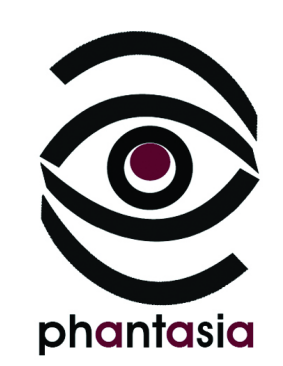

\title{
Le langage au pouvoir. Foucault, lecteur de Brisset
}

\author{
Philippe Sabot \\ CNRS, UMR 8163 - STL - Savoirs Textes Langage, Université de Lille
}

L'exception infirme la règle ${ }^{1}$

La réflexion qui suit porte pour l'essentiel sur le bref texte que Foucault a consacré à Jean-Pierre Brisset et qui s'intitule : «Sept propos sur le Septième ange ». Pourquoi ce texte au titre à première vue énigmatique retient-il l'attention? Il ne s'agit après tout que d'une courte préface (en sept points dont un de présentation bio-bibliographique) à deux ouvrages de Jean-Pierre Brisset, La Grammaire logique (publié en 1878$)^{2}$ et $L a$ Science de Dieu ou la Création de l'Homme (ouvrage publié en 1900 par l'éditeur Chamuel) réédités par l'éditeur Claude Tchou en $1970^{3}$. Ajoutons qu'au moment où Foucault s'y est intéressé, à partir du début des années $1960^{4}$, Brisset passait, tout comme Roussel d'ailleurs, pour l'un de ces excentriques, l'un de ces «fous littéraires » auquel Breton avait consacré en 1940 un chapitre de son Anthologie de l'humour noir. Dans ce chapitre (comportant une notice et des extraits de La Science de Dieu), Breton note ainsi qu' ' il est frappant que l'œuvre de Raymond Roussel, l'œuvre littéraire de Marcel Duchamp, se soient produites, à leur insu ou non, en connexion étroite avec celle de Brisset, dont l'empire s'est peut-être étendu jusqu'aux essais les plus récents de dislocation poétique du langage $»^{5}$. Étaient alors cités, parmi ces essais les plus remarquables, ceux de Léon-Paul Fargue, de Robert Desnos, de Michel Leiris, d'Henri Michaux et de James Joyce. C'est peut-être d'ailleurs directement dans cette Anthologie, ou bien encore dans un bref article de

\footnotetext{
${ }^{1}$ Cette formule figure sur la page de couverture de la première édition de La Grammaire logique (1878). Voir infra note 9.

${ }^{2}$ Le titre complet de l'ouvrage de Jean-Pierre Brisset, publié en 1878 par la Librairie de J.-B. de Fruchy, est $L a$ Grammaire logique, ou théorie d'une nouvelle analyse mathématique résolvant les questions les plus difficiles et traitant à fond : $1^{\circ}$ du participe passé ; $2^{\circ}$ du participe présent ; $3^{\circ}$ du placement des pronoms après l'impératif; en tout, trois règles logiques, les seules vraies et sans aucune exception, résumées en douze mots. Lors de la réédition de l'ouvrage en 1883 chez l'éditeur Ernest Leroux, le titre évolue mais réaffirme la vocation universelle du propos : $L a$ Grammaire logique, résolvant toutes les difficultés et faisant connaître par l'analyse de la parole la formation des langues et celle du genre humain.

${ }^{3}$ FoucAult (M.), «Sept propos sur le septième ange », préface à Jean-Pierre Brisset, La Grammaire logique et La Science de Dieu, Paris, Tchou, 1970, p. VII-XIX. Ce texte a été repris dans les Dits et écrits (Paris, Gallimard, «Bibliothèque des sciences humaines », 1984, vol. II, texte $\mathrm{n}^{\circ} 73$, p. 13-25; ensuite cité $D E$, suivi des numéros de volume, de texte et de la pagination) ainsi que dans un opuscule publié en 1986 chez Fata Morgana. Dans la suite de ce chapitre, nous citerons par commodité la préface de Foucault d'après cette dernière édition, abrégée en Sept propos..., suivi de la pagination. Nous citerons les textes de Brisset d'après l'édition Tchou de 1970. Signalons pour être complet qu'une édition des Euvres complètes de Brisset a été proposée par Marc Décimo en 2001, aux Presses du réel (collection « L'écart absolu »).

${ }^{4}$ Un premier texte, intitulé « Le cycle des grenouilles », paraît en 1962 dans la Nouvelle Revue Française $\left(10^{\mathrm{e}}\right.$ année, $\mathrm{n}^{\circ} 114$, juin 1962, p. 1159-1160). Il porte sur La Science de Dieu. Ce texte est repris in DE, I, n 9, p. 203-205.

${ }^{5}$ Breton (A.), Anthologie de l'humour noir, Paris, Éditions du Sagittaire, 1940 ; rééd. Le Livre de poche, 1973 , p. 235.
} 
Queneau paru en avril 1956 dans la revue Bizarre ( La Théologie génétique de Brisset » ${ }^{6}$ ), que Foucault a pris connaissance du travail à la fois monumental et proprement inclassable de celui qui se présentait sur ses cartes de visite comme «le septième Ange de l'Apocalypse et l'Archange de la Résurrection ${ }^{7}$ et dont le principal titre de gloire est qu'il avait été élu «Prince des penseurs » en 1913 (recueillant plus de 200 voix sur 300, alors que M. Bergson, pourtant membre de l'Institut, n'en recueillait que $55 \ldots$ ). Terminons cette présentation générale en soulignant que la carrière de Brisset diffère profondément de celle de Roussel, écrivain méconnu lui aussi, mais véritable obsédé de littérature, dans la mesure où elle s'égrène comme un inventaire à la Prévert ou comme une encyclopédie chinoise à la Borgès : Brisset fut en effet tour à tour apprenti-pâtissier, soldat (aux campagnes de Crimée, d'Italie et d'Allemagne), maître-nageur, officier de police judiciaire, professeur de langues vivantes ${ }^{8}$, grammairien, commissaire de surveillance administrative aux Chemins de fer...

Nous devons nous demander alors, au-delà de ce fourmillement des étiquettes professionnelles, aussi désarçonnantes qu'amusantes par la série improbable qu'elle représente, quelle est la figure de Brisset qui retient précisément l'attention de Foucault et qui oriente sa première approche d'une œuvre pour le moins difficile à appréhender comme une totalité stable. Il semble évident de ce point de vue que l'expérienceBrisset entretient une parenté puissante avec l'expérience-Roussel ${ }^{9}$ et que la préface de 1970 constitue pour une part un prolongement de l'ouvrage de 1963, comme en témoigne l'intérêt porté au " procédé » de Brisset comparé à celui de Roussel (et à celui de Wolfson). Mais il est indéniable également que les " sept propos » de 1970 ne forment pas seulement une réplique du livre consacré à Roussel, et notamment parce que justement ces procédés diffèrent et que la production d'écriture qui en découle est d'une tout autre nature chez l'un et chez l'autre. Il importe en effet de relever que la lecture que Foucault propose des fantaisies de Brisset s'oriente résolument vers la réflexion hétérodoxe que ce dernier, qui avait des prétentions de grammairien plus que de littérateur, propose au sujet de l'origine des langues, prenant en particulier à revers les principes théoriques d'un certain savoir linguistique. Enfin, il faut se demander si la modeste préface consacrée à Brisset ne mérite pas d'être située elle-même à cette charnière du travail de Foucault qui envisage notamment les rapports du discours et du pouvoir (dans la leçon inaugurale au Collège de France, L'ordre du discours et dans les premières Leçons sur la volonté de savoir, contemporaines de la préface publiée chez Tchou). Il est possible que cette réorientation politique du propos relève d'un intérêt de plus en plus marqué pour des usages dérangeants, dissonants, du langage, venant contrarier le déploiement ordonné du symbolique et la prise de pouvoir du langage sur nos vies et nos êtres. Ce qui surgit alors au cœur de l'analyse, c'est le pouvoir du langage, à entendre comme sa puissance d'analyse des rapports de pouvoir.

\section{Brisset, Roussel, Wolfson ou le langage en délire}

Il nous semble qu'il n'est pas besoin de savoir plusieurs langues pour sentir combien notre analyse des mots est juste ; que toutes les langues ont bien leur origine dans les premiers cris que poussèrent les êtres appelés à former le genre humain ${ }^{10}$.

Reprenons les choses depuis le début et repartons de cette espèce de dispositif Roussel-Brisset tel qu'il semble se mettre en place et fonctionner chez Foucault. Il faut insister tout d'abord sur le fait que, si les pratiques d'écriture de Roussel et de Brisset peuvent commodément être rangées dans la catégorie (un peu fourre-tout) des «fous littéraires ${ }^{11}$, ce n'est pas sous cet angle de l'expression de leur supposée «folie»

\footnotetext{
${ }^{6}$ Cet article ainsi que la plupart des textes se rapportant à Brisset sont recueillis dans la somme que Marc Décimo a consacrée à Jean-Pierre Brisset. Prince des penseurs, inventeur, grammairien et prophète (Paris, Les Presses du Réel, « L’écart absolu », 2001).

${ }^{7}$ Voir DÉCIMO (M.), op. cit., p. 534.

${ }^{8}$ C'est ainsi que Brisset se présente lui-même sur la page de couverture de la première édition de La Grammaire logique: "Professeur de langues vivantes, auteur d'une Nouvelle Méthode à l'usage des allemands ». Et il est ajouté juste au-dessous de cette présentation : «L'exception infirme la règle ». Cet ajout deviendra : «La Parole est Dieu » dans la seconde édition, cinq ans plus tard.

9 Au sujet de l'«expérience-Roussel», nous renvoyons à la notice introductive du Raymond Roussel [1963], in Foucault (M.), Euvres, Paris, Gallimard, « Bibliothèque de la Pléiade », 2015, I, p. 1552-1562.

${ }^{10}$ BRISSET (J.-P.), La Grammaire logique, op. cit., p. 120.

11 Sur cette catégorie de «fous littéraires », voir l'article synthétique de Jean-Jacques Lecercle, in Encyclopaedia Universalis [en ligne], consulté le 25 juillet 2018. URL: http:/www.universalis.fr/encyclopedie/fous-litteraires/.
} 
dans le langage que Foucault s'intéresse à leurs productions, mais il s'y rapporte plutôt en tant que les tours et les détours de leur pratique langagière les conduit à faire " délirer » le langage et l'ordre du discours qui en contrôle d'ordinaire le cours et les productions.

Mais le délire n'est pas du même ordre ici et là. En effet, au délire de Roussel qui le conduit à inventer de toutes pièces, mais toujours avec les pièces d'un langage donné qu'il se plaît à détraquer, des univers improbables et des récits fantastiques peuplés de créatures et de machineries étranges, répond le délire de Brisset, fondé sur une formidable inventivité grammaticale, lexicale et sémantique et surtout sur le principe en apparence très sérieux d'une recherche sur l'histoire de la formation des mots, dont la connaissance nous ouvrirait directement à la connaissance des choses. Le délire de Roussel est, si l'on veut, interne à l'exercice de la littérature, même s'il a une portée ontologique (il fait communiquer le langage et la mort à travers la fiction $^{12}$ ); celui de Brisset n'est pas d'abord d'ordre littéraire (il n'est en ce sens un écrivain que par défaut), mais il appartient - ou du moins il revendique une appartenance - au registre de la linguistique, même si ses " recherches" reposent sur une confusion permanente entre linguistique et histoire, entre grammaire et cosmogonie, entre étymologie et mythologie, entre fiction et logique. Il est possible de dire que, pour Foucault, l'intérêt de Brisset tient à cette manière, à la fois ingénue et sophistiquée, qu'il a de faire naître une écriture d'un savoir poussé jusqu'au délire, d'entretenir rigoureusement la confusion entre différents usages des mots et du langage pour en faire même l'essentiel d'une méthode d'investigation dont la valeur scientifique lui apparaissait tout à fait évidente : car il était persuadé que son enquête sur l'origine des langues allait « changer complètement l'étude de la linguistique » ${ }^{13}$.

De quoi parle-t-on exactement? D'une enquête d'allure étymologique qui affirme que le langage et l'être communiquent en profondeur dans la mesure où ils sont au contact d'une origine perdue, cette origine que l'enquête de Brisset vise précisément à retrouver, quitte à l'inventer, quitte à la reconstituer par bribes ou par décomposition-recomposition du langage qui est à notre disposition :

[Nous avons] démontré avec une évidence inéluctable la création de l'homme; que l'histoire de cette création [a] été écrite et scellée dans la bouche de l'homme, sans qu'il en eût le moindre soupçon ; qu'il [a] oublié son origine tout en en ayant l'historique dans les fables mythologiques de tous les pays, dans la Bible et dans le langage journalier ${ }^{14}$.

Une origine a eu lieu et c'est l'oubli de cette origine (et de son lieu, le langage) qu'il faut réparer en allant chercher dans la bouche de l'homme ce qui s'y trouve scellé malgré lui. Dans sa préface, Foucault lie à cette «évidence inéluctable» dont parle Brisset deux principes complémentaires qui guident la recherche de celui-ci et qui permettent de fixer le profil délirant de cette recherche : le principe dit de «non traduction » et le principe de prolifération interne.

Le premier principe contrevient à l'idée même d'une quête de l'origine des langues, c'est-à-dire à la recherche de ce " petit ensemble d'éléments simples liés aux choses et demeurés sous forme de traces dans toutes les langues du monde ${ }^{15}$. Là où les recherches sur l'origine des langues (depuis la Renaissance, avec Claude Duret puis à l'âge classique avec De Brosses ou Court de Gébelin) ${ }^{16}$ tendaient à circonscrire un idiome primitif, proche du cri, et les lois élémentaires de construction à partir desquels il est possible ensuite de dériver toutes les langues par un jeu de complications progressives et successives, la démarche de Brisset mime et raille leur scientificité. Il s'en tient strictement à la langue actuelle en la dotant, par une décision parfaitement arbitraire, d'une potentialité insoupçonnée et, surtout, d'une capacité d'éclaircissement des

Wolfson fait ici figure de pièce rapportée. La publication de son ouvrage Le Schizo et les langues dans la collection «Connaissance de l'inconscient» de J.-B. Pontalis a fortement influé sur sa réception, largement dominée par une littérature analytique et ce, même si un grand nombre de commentateurs s'efforcent de souligner l'originalité profonde du livre de Wolfson qui échappe aux codes de la «Littérature » et qui ne peut se réduire non plus à la documentation d'un cas clinique. Voir à ce sujet les éléments de bibliographie établis par Maria Eugenia Uriburu à la fin du Dossier Wolfson ou l'affaire du Schizo et les langues (Paris, Gallimard, « L'arbalète », 2009).

${ }^{12}$ Sur cette communication du langage et de la mort chez Roussel, voir notre introduction à Raymond Roussel [1963], in Foucault (M.), Euvres, I, en particulier p. 1559-1560.

${ }^{13}$ BRISSET (J.-P.), La Grammaire logique, op. cit., p. 90-91.

${ }^{14}$ BRISSET (J.-P.), La Science de Dieu, op. cit., p. 225.

${ }_{15}$ Sept propos..., p. 11-12.

${ }^{16}$ Rappelons que Michel Foucault consacre, dans Les Mots et les choses, toute une étude à ces théories de l'origine du langage, de la Renaissance à l'âge classique. Voir Les Mots et les choses. Une archéologie des sciences humaines [1966], in Foucault (M.), Euvres, I, p. 1082-1084 (sur Claude Duret et son Trésor de l'histoire des langues [1613]), p. 1156-1162 (sur la théorie des racines à l'âge classique). 
origines conjointes de la langue et de l'homme - l'homme n'étant au fond qu'un jeu de mots ou même que le bruit primitif d'une langue en décomposition-recomposition permanente : un gargouillement dans une mare infâme, pleine de « saloperies ».

De cette manière, le «Prince des penseurs » procède selon Foucault à une requalification radicale de 1' " origine » qui n'est pas située dans une extériorité ou dans une antériorité à la langue actuelle (le français), et par rapport à laquelle celle-ci serait seconde et dérivée : Brisset dit lui-même que « l'origine de chaque langue est dans cette langue même ${ }^{17}$. Du principe de l'unité et de la fixité de la parole humaine (qui ne peut varier), il s'ensuit que les langues ne sauraient évoluer les unes dans les autres : chaque langue était à l'origine ce qu'elle est maintenant; à chaque instant, l'origine se répète, elle a de nouveau lieu. Donc, le français n'est manifestement pas dérivé du latin: «La langue latine ne nous paraît pas avoir formé le français $»^{18}$. Mieux : Brisset est fier, nous dit Foucault, d'avoir démontré que le latin n'existait pas ${ }^{19}$ ! Ce n'est, à en croire l'auteur de La Grammaire logique, qu'une sorte d'argot italien inventé au XVIII ${ }^{\mathrm{e}}$ siècle par un groupe de « brigands » romains qui voulaient se forger un "langage spécial, facile à apprendre » qu'ils fussent les seuls à comprendre pour mener leurs petites affaires en secret ${ }^{20}$. Précisons encore l'enjeu de cette affaire : «Le latin est artificiel $»^{21}$. Si le latin existait (vraiment), rien ne serait permis : la langue primitive serait compacte et fondamentale, irréductible, elle prescrirait à notre langage ses formes de dérivation, ses règles et ses usages. Alors que, libéré de cet élément premier censé fixer la loi de tout langage, le mot explose successivement « en plusieurs combinaisons élémentaires, si bien que sa forme actuelle découvre, lorsqu'on la décompose, plusieurs états archaïques. [...] C'est à la science de Dieu de les faire réapparaître et tourner comme un grand anneau multicolore autour du mot analysé $»^{22}$. L'archaïque déjoue l'originaire et le rejoue sur la scène de notre langage : celui-ci est le même, et en même temps, il est toujours différent. Second principe : la prolifération.

À l'origine, pour Brisset, il y a donc cette langue, notre langue «à l'état de jeu ", un "langage en émulsion ${ }^{23}$ qui livre sa propre origine instable lorsqu'on le parcourt et qu'on le répète un peu au hasard ou en s'autorisant des approximations révélatrices, lorsqu'on se met à y circuler librement pour en explorer les coins et les recoins, sans retenue et en se fiant avant tout aux possibilités illimités qu'il recèle pour qui sait le manipuler et extraire la polyphonie de ses origines. L'étymologie fantastique de Brisset révèle ici sa véritable nature poïétique, inhérente à son procédé, tel que Foucault propose de l'expliciter. Jouer avec le langage, c'est pour l'auteur de la Grammaire logique, faire défiler le signifiant, le laisser se défiler plutôt que l'assigner à un signifié fixe, le laisser dériver, non à partir d'une origine, mais en roue libre. Sous la plume de Brisset, les mots prennent la tangente et explosent en plein vol : les sons dont se compose un mot appellent, par ressemblance, par contiguïté ou contagion, d'autres mots dont les sons, à leur tour, vont évoquer d'autres mots, etc. Il se produit ainsi une chaîne d'équivalences homonymiques ou paronymiques qui, simultanément, fonctionne comme chaîne synonymique, comme chaîne d'équivalences sur le plan du signifié :

Voici les salauds pris. Ils sont dans la sale eau pris, dans la salle aux prix. Les pris étaient les prisonniers que l'on devait égorger. En attendant le jour des pris, qui était aussi celui des prix, on les enfermait dans une salle, une eau sale, où on leur jetait des saloperies. Là on les insultait, on les appelait salauds. Le pris avait du prix. On le dévorait et, pour tendre un piège, on offrait du pris et du prix : c'est du prix. - C'est duperie, répondait le sage, n'accepte pas de prix, ô homme, c'est duperie ${ }^{24}$.

Ce procédé de décomposition et d'analyse du langage (saloperie-duperie) semble très proche de celui dont parle Roussel dans Comment j'ai écrit certains de mes livres, du moins dans sa forme évoluée : « Je fus conduit à prendre une phrase quelconque dont je tirais des images en la disloquant, un peu comme s'il se fût agi d'en extraire des dessins de rébus ». Et Roussel de donner comme exemple de ce procédé, la transformation suivante qui forme la matrice sémantique du conte Le Poète et la Moresque : «J'ai du bon tabac dans ma tabatière/Jade tube once aubade en mat a basse tierce $»^{25}$. Comme le note Foucault à la fin de

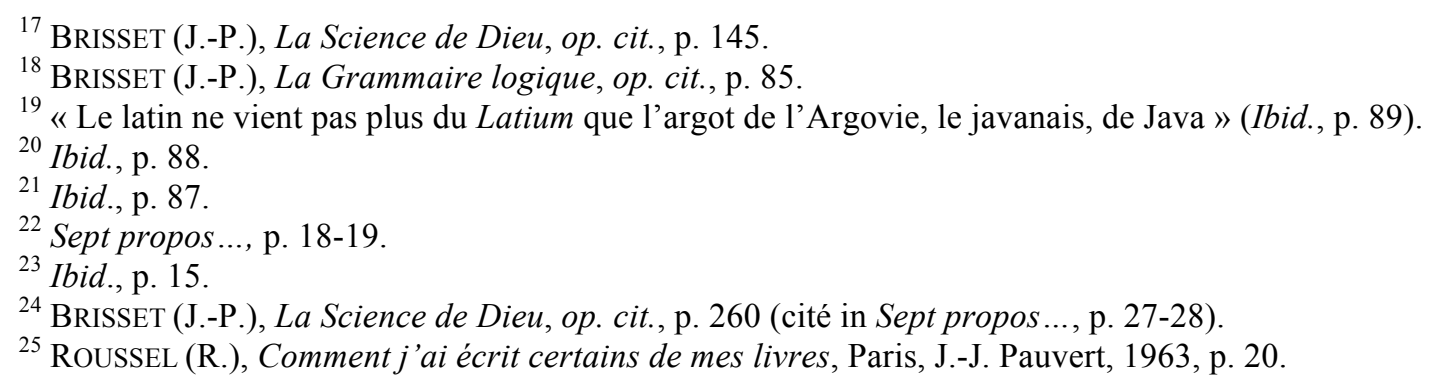


sa préface, la dislocation roussellienne fait surgir au sein même de notre langage familier les figures les plus étranges et les scènes les plus merveilleuses, avec lesquelles « les mots font corps ${ }^{26}$. Il reste que ce corps-àcorps avec le langage, qui le blesse et l'ouvre sur lui-même, débouche sur la conjuration du hasard premier de la langue qui recèle toujours un silence de mort. Parler, écrire contre la mort qui rôde : le procédé roussellien est lesté par cette inquiétude sourde qui renvoie le langage à la mort et à la disparition élocutoire de celui qui parle. L'espace ouvert entre les mots se referme comme une tombe sur le cadavre de l'auteur.

Or, l'opération que propose Brisset est d'un tout autre ordre. Au lieu d'écarter le langage de lui-même, de creuser ses fissures phonologiques pour prendre la mesure de $l^{\prime}$ " abîme presque infranchissable » ${ }^{27}$ qui se loge entre chaque son, entre chaque mot et dans chaque phrase, même la plus anodine, il s'agit de sauter d'un mot à l'autre, de se laisser emporter par les torsions du son qui requalifient le sens des mots et « font surgir à chaque fois tout le bariolage d'une scène nouvelle. Autour du son, les scènes tournent comme à la périphérie d'une grande roue $»^{28}$. La Grammaire logique de Brisset, c'est la foire des mots, la foire aux mots; et il n'y a au fond qu'à circuler au milieu de ces histoires assemblées par hasard mais qui finissent par dire quelque chose du hasard fondamental qui est lié à l'origine commune du langage et des hommes. Hasard que recouvrent depuis si longtemps d'un voile d'oubli les proférations ordinaires et les recherches savantes des linguistes qui ont hâte de le convertir en nécessité. Pour reprendre l'exemple cité par Foucault : il convient de ne pas «réduire la distance entre saloperie et duperie, pour rendre vraisemblable qu'on ait pu la franchir », mais de l'accuser en éloignant ces mots l'un de l'autre, et d' « hérisse[r] l'espace qui les sépare d'événements divers, de figures improbables et hétérogènes ", de le "peuple[r] du plus grand nombre de différences possible $»^{29}$ qui seront autant de scènes fictives, évoquées dans un clignotement verbal et mental indéfini (en droit) dont le phonème [pri] constitue la matrice élémentaire, le ressort d'une dissociation possible. Ainsi, la seule unité qui demeure, hors de toute référence, est due au fait que «malgré la diversité du décor, des acteurs, des péripéties, c'est le même bruit qui court, le même geste sonore qui se détache de la mêlée et flotte un instant au-dessus de l'épisode, comme son enseigne audible ${ }^{30}$. Roussel cherche à conjurer le hasard de naissance du langage en tant qu'il forme le masque d'une mort certaine. Brisset fait de ce hasard l'équivalent d'une origine qui, au lieu de constituer une référence ultime et simple d'où dériverait la complexité et la pluralité des langues, n'en finit pas de se dire ; qui se fait être en se disant, qui se démultiplie dans les modulations d'un bruit de fond ininterrompu. A l'origine, était le multiple.

Cette multiplicité bruissante, ce «vacarme primitif ${ }^{31}$ qui retient l'attention de Foucault chez Brisset l'éloigne aussi de Wolfson et de son procédé schizophrénique de traduction continue et instantanée qui vise avant tout à conjurer la «mauvaise matière malade ${ }^{32}$, à neutraliser le langage, sa propre langue, sa langue maternelle en la faisant passer hors d'elle-même, en la retournant dans d'autres idiomes où les mots s'apaisent - au moins ou seulement de manière provisoire (car c'est une lutte sans fin). Le rejet d'un idiome originaire n'a pas le même sens que celui de la langue maternelle pour Wolfson. En effet, pour Brisset, l'origine n'est pas une mais multiple: il faut la faire proliférer dans le langage pour en recueillir la constellation imaginaire. Origine et imagination sont, pour cette raison, apparentées :

Voici la double étymologie - et admirons justement la double gémellité - d'origine et d'imagination : «Eau rit, ore ist, oris. J'is næud, gine. Oris gine = la gine urine, l'eau rit gine. Au rige ist noud. Origine. L'écoulement de l'eau est à l'origine de la parole. L'inversion de oris est rio, et rio ou rit eau, c'est le ruisseau. Quant au mot gine il s'applique bientôt à la femelle : tu te limes à gine ? Tu te l'imagines. Je me lime, à gine est ? Je me l'imaginais. On ce, l'image ist né ; on ce, lime a gine ai, on se l'imaginait. Lime a gine, à sillon ; l'image ist, næud à sillon ; l'image ist, n'ai à sillon $»^{33}$.

Pour Wolfson, l'origine est loin d'avoir cette fluidité et cette évanescence jouissive que lui attribue Brisset dans le ruissellement des choses dites. Elle a plutôt la dureté et l'intangibilité d'un rappel à l'ordre,

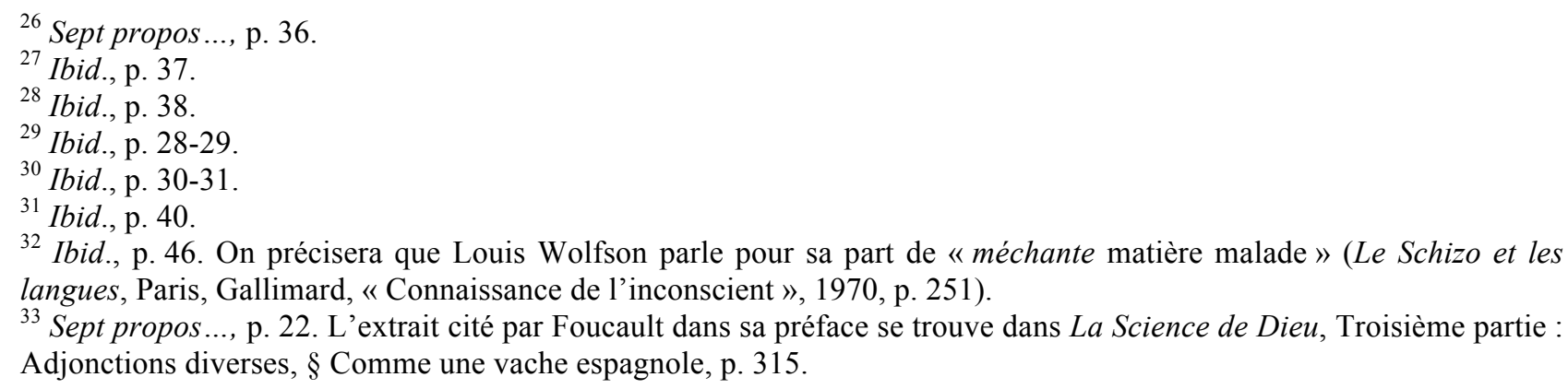


elle dessine la limite du langage, là où les mots se nouent au corps et le menacent littéralement d'asphyxie, en l'entraînant dans leur pente néfaste, celle qui ramène à la mère.

Par ailleurs, comme le souligne Deleuze dans sa préface au Schizo et les langues, ce qui sépare également le procédé de Wolfson de celui de Roussel, ce n'est pas seulement la manière dont il tord le langage dans ses traductions délirantes (non pas vers une origine mais contre toute origine, pour conjurer l'origine ellemême), mais c'est surtout que ces «transformations linguistiques ne dégagent aucun événement pur idéel ayant une existence esthétique, mais restent entièrement subordonnées aux accidents dans lesquels la phrase maternelle réelle a été prononcée, et la transformation imaginaire, effectuée ${ }^{34}$. Le registre dans lequel s'inscrit le procédé de Wolfson n'est ni celui de la science ni celui de l'art : ou alors il est leur simulacre, leur version ironique, qui convertit la douleur et l'angoisse de la fêlure intime en une étude «imbécilique ${ }^{35}$ et joyeuse de l'infinie variété des langues et des ressources folles qu'elles mettent à notre disposition pour en conjurer la nocivité. La langue comme simulacre, puissance du faux au service d'une angoisse, plutôt que comme terrain de jeu et surface lisse d'un délire sans attaches.

\section{Le langage et la scène du pouvoir}

Bruit unique. Saloperie des guerres, et des victoires dans la boue. Saloperie de la foule en fête injuriant les captifs. Saloperie des prisons. Saloperie des marchés où s'achète la viande des hommes ${ }^{36}$.

Il est cependant un autre aspect de la brève étude de Foucault sur Brisset qui mérite d'être relevé et qui permet aussi d'inscrire ce texte dans une autre séquence du travail de Foucault sur la littérature et les écritures en délire.

C'est que, si toute syllabe renvoie à une idée, tout mot renvoie à un complexe d'idées, c'est-à-dire à une phrase qui exprime (en ce sens qu'elle la constitue autant qu'elle la dénote) une "scène ». Avec Brisset, le langage devient en quelque sorte la représentation du Drame des origines. Comme nous l'avons vu, il s'agit d'une représentation interminable qui a lieu de nouveau à chaque fois que nous parlons. Or, en dressant sous nos yeux, cette scène fantastique d'une fragmentation-recomposition du langage, scène d'origine et scène d'imagination à la fois, Brisset ne cherche pas seulement à nous communiquer la jubilation du savant qui fait part de ses découvertes. Du moins Foucault insiste-t-il sur une autre dimension de ces histoires de mots à dormir debout qui ne racontent rien d'autre que la perpétuelle renaissance du langage, de notre langage, si familier et pourtant si étrange - et même si inquiétant dès que le grammairien s'en empare pour le reconstituer et l'assigner à ses propres lois.

À nouveau donc, l'inquiétude. Mais ce n'est pas l'inquiétude qui forme l'envers sombre des contes et légendes de Roussel et constitue le reflet de cette finitude interne et néanmoins fondamentale creusant le langage de sa puissance de mort. C'est plutôt cette fois l'inquiétude de ce bruit qui court tout au long d'une histoire sans fin et sans origine, d'une histoire des hommes et des mots qui les forment et qui les font vivre et parler. C'est l'inquiétude qui jaillit des choses dites, de leur bruissement et de leur ruissellement hors de tout contrôle, ou du moins hors du contrôle définitif que leur imposerait un «ordre du discours ». Nous n'avons plus affaire à l'extériorité neutre des énoncés que prétend décrire - et maintenir - L'Archéologie du savoir ${ }^{37}$. Il faut plutôt reprendre ici, en écho à cette inquiétude, la question par laquelle s'ouvre la leçon inaugurale au Collège de France (prononcée, rappelons-le, quelques semaines à peine après la sortie des œuvres de Brisset chez Tchou, préfacées par Foucault) : " Mais qu'y a-t-il donc de si périlleux dans le fait que les gens parlent, et que leurs discours indéfiniment prolifèrent? Où donc est le danger ? $»^{38}$.

Or, ce danger est clairement identifié par Foucault dans le délire de Brisset. Ses scènes de langage « forment une histoire ${ }^{39}$ et cette histoire, déliée de toute fondation originaire (c'est-à-dire du rapport à une Ursprung [origine] d'où jaillirait le sens premier des mots et à laquelle s'accrocherait la " genèse lente » de

\footnotetext{
${ }^{34}$ Deleuze (G.), « Schizologie », Préface à Louis Wolfson, Le Schizo et les langues, p. 8.

35 «Qu'il était agréable d'étudier les langues, même à sa manière folle, sinon imbécilique ! (WoLFSON (L.), Le Schizo et les langues, p. 70)

${ }^{36}$ Sept $\operatorname{propos} \ldots$, p. 33.

${ }^{37}$ Foucault (M.), L'Archéologie du savoir, Paris, Gallimard, « Bibliothèque des sciences humaines », 1969.

${ }^{38}$ Foucault (M.), L'Ordre du discours, Paris, Gallimard, 1971, p. 10.

${ }^{39}$ Sept propos..., p. 31.
} 
leur formation), retrace de manière chaotique et ouverte, dans la forme d'une reprise perpétuelle et de nouvelles scénographies verbales, «comment des discours pris dans des scènes, dans des luttes, dans le jeu incessant des appétits et des violences, forment peu à peu ce grand bruit répétitif qui est le mot, en chair et en os $»^{40}$. L'histoire, les histoires au pluriel, que nous raconte Brisset ne sont donc pas des retours à l'origine ni la pure et simple restitution d'une origine des langues. Son délire le conduit ailleurs, dans la dimension de ce que Foucault au même moment analyse avec Nietzsche, comme une "généalogie ». Il reconduit en effet l'origine elle-même à sa provenance (Herkunft) corporelle et sexuelle, aux débats du corps avec lui-même et avec ce flux incessant des choses dites. Il la reconduit aussi à la loi d'une " émergence » (Entstehung) qui dit « le principe et la loi singulière d'une apparition ${ }^{41}$, mais de cette sorte d'apparition qui est aussitôt menacée de disparition puisqu'elle est inscrite d'emblée dans un rapport de forces, dans une lutte à l'issue incertaine. Pour Foucault, d'après Brisset, lui-même relu par-dessus l'épaule de Nietzsche, c'est le langage tout entier qui est le fruit et l'objet d'une lutte, et d'une lutte continue. Les mots que nous utilisons pour désigner les choses sont les dépôts, les indices, les symptômes matériels et provisoires de cette lutte.

À y regarder de près d'ailleurs, comme le fait Brisset, c'est bien cette lutte qu'ils désignent plus que le monde sur lequel ils se posent et avec lequel ils composent une illusoire unité. Le délire linguistique, parce qu'il contourne l'origine des langues, ne nous fait pas accéder à la certitude du sens et à la transparence d'une représentation articulée et stabilisée. Il nous donne à entendre à nouveau le bruit de la bataille, le tumulte des origines et la violence des affrontements qui précèdent les désignations et l'ordre du discours. Voici comment Foucault caractérise alors en ce sens l'opération propre à Brisset :

\begin{abstract}
Autour d'un mot quelconque de sa langue, [...] il convoque, à grands cris allitératifs, d'autres mots, dont chacun traîne derrière lui les vieilles scènes immémoriales du désir, de la guerre, de la sauvagerie, de la dévastation [...]. Il entreprend de restituer les mots aux bruits qui les ont fait naître, et de mettre en scène les gestes, les assauts, les violences dont ils forment comme le blason maintenant silencieux. Rendre le Thesaurus linguae gallicae au vacarme primitif; retransformer les mots en théâtre ; replacer les sons dans ces gorges coassantes ; les mêler à nouveau à tous ces lambeaux de chair arrachés et dévorés... ${ }^{42}$.
\end{abstract}

Aux jubilations innocentes des scènes de foire ou des bestiaires fantastiques (les hommes-grenouilles) se superpose ainsi la violence inattendue de scènes de guerre, qui mettent le langage en contact avec le désir, avec ces cris et ces gestes primitifs par où il fait corps avec celui qui parle et qui prend le pouvoir, ou le perd, dans et par cette parole proférée.

La Grammaire logique de Brisset redonne le pouvoir au langage (plutôt qu'à celui qui l'énonce), mais, ce faisant, elle dénonce aussi le rapport entre langage et vérité sur lequel se fondent l'ordre du discours et la maîtrise des sujets parlants sur leurs énoncés. Dès lors que la langue se décompose d'elle-même et amorce sa propre généalogie, elle rejoint cette dimension d'événementialité qui porte sa puissance et inquiète ceux qui souhaitent assurer leur prise sur le discours pour en tirer parti. Les généalogies fantastiques de Brisset événementialisent le langage en le brisant pour le recomposer, mais jamais à l'identique puisque ces recompositions le lestent d'un bruit sédimentaire qui rend désormais les mots instables et qui en ruine toute signification univoque. L' « origine », une fois passée au crible de l'analyse brissetienne, s'entend elle-même comme un ruissellement sans commencement ni fin ; elle est ainsi libérée du sens (unique) qu'elle pouvait recevoir tant que son étymologie n'avait pas révélé sa troublante parenté avec l'imagination et avec les puissances bestiales du désir sexuel.

Mais surtout, cette événementialisation apporte une dimension politique nouvelle à l'histoire, aux histoires du langage que propose Brisset. Celles-ci fournissent en effet, nous dit Foucault, " un décor pour rejouer des scènes de violence, de meurtre et d'anthropophagie ${ }^{43}$. Or, c'est bien dans ce décor, sur cette scène (et non dans la direction d'une scène originaire) que se joue le drame d'CEdipe auquel Foucault consacre, on le sait, un certain nombre d'interventions au tout début des années 1970, que ce soit dans ses premiers cours au Collège de France ou dans d'autres contextes ${ }^{44}$. Ce drame, c'est celui d'une vérité qui

\footnotetext{
${ }^{40}$ Ibid., p. 34.

${ }^{41}$ Foucault (M.), « Nietzsche, la généalogie, l’histoire » [1971], in DE, II, n 84, p. 143.

${ }^{42}$ Sept propos..., p. 40-41.

${ }^{43}$ Ibid., p. 47.

${ }^{44}$ Voir notamment les Leçons sur la volonté de savoir. Cours au Collège de France 1970-1971 (Paris, Gallimard-Le Seuil, «Hautes Études», 2011, Leçon du 17 mars 1971 et son développement dans la conférence "Le savoir d'EEdipe », p. 225-251) et « La vérité et les formes juridiques » [1973], in DE, II, n 139, p. 553sq. Sur l'importance de
} 
échappe à celui qui la cherche ; c'est celui d'un discours qui manque la vérité et qui contraint le sujet à la recevoir des autres - ce qui le destitue de son propre pouvoir (sur lui-même et sur les autres).

Mais plus largement, le décor que plante Brisset avec ses étymologies fantastiques et le «tourbillon de [ses] scènes frénétiques, sauvages ou jubilatoires, d'où les mots surgissent et que les mots appellent ${ }^{45}$, c'est celui dans lequel Foucault lui-même inscrit sa propre recherche sur le discours philosophique, notamment lorsque, à l'ouverture des premières leçons au Collège de France, il s'interroge sur le partage " originaire » du discours philosophique et de la sophistique. Ce partage concerne les procédures et les conditions d'attribution d'une valeur de vérité à un énoncé. Du point de vue du discours philosophique, il importe que l'énoncé soit intégré de manière réglée (et anonyme) à un jeu de propositions qui définissent entre elles un rapport de signification et qui définissent par rapport aux choses un rapport de désignation stable. Les règles du discours et l'articulation du signifiant à un signifié-référent garantissent sa valeur de vérité. Or, du côté du discours sophistique, c'est bien plutôt «l'existence d'un énoncé » qui s'impose, non pas tant de lui-même, que dans le jeu d'une reprise et d'un réagencement toujours possibles au gré de la discussion, voire de la polémique :

Le sophisme prend appui [...] sur l'existence d'un énoncé ; sur le fait que des mots ont été prononcés et qu'ils demeurent là, au centre de la discussion, comme ayant été produits et pouvant être répétés, recombinés au gré des partenaires ; c'est dit, c'est dit : non point comme une forme idéale, régulière et qui peut recevoir certains types de contenu, mais un peu comme ces trophées que les guerriers après la bataille mettent au milieu d'eux et qu'ils vont s'attribuer, non sans dispute et contestation eis meson ${ }^{46}$.

Avec le sophisme, les sujets se saisissent des événements discursifs au point de faire corps avec eux et ils entrent ainsi dans une guerre de position où la matérialité du discours devient l'enjeu d'une lutte, d'un investissement subjectif et corporel (de l'ordre de l'incorporation) qui fait prévaloir le rapport de forces.

Selon Foucault, Brisset offre ainsi, aux côtés de Wolfson et de Roussel, et en tout cas du côté d'une expérience du langage irréductible à l'ordre du discours, une réplique de cette événementialisation de l'énoncé que les sophistes avaient initié en confondant les jeux du langage, les jeux de la vérité et les jeux du pouvoir: "Les vrais sophistes aujourd'hui ne sont peut-être pas les logiciens mais Roussel, Brisset, Wolfson ${ }^{47}$. Il est clair qu'en restituant le langage à la matérialité d'une chose dite et en réinscrivant cette matérialité dans la dimension d'une histoire mouvementée, aimantée par les jeux du pouvoir et du désir, Brisset a durablement perverti les recherches sur l'origine des langues. Son délire fait entendre et résonner la voix d'une autre logique du discours, d'une autre logique du sens qui s'écrit dans les marges de la philosophie - à moins que la philosophie elle-même ne finisse par s'écrire elle-même dans sa propre marge, en se mettant à l'écoute de ces cris coassants et de ces gestes inchoatifs qui décentrent la parole et le sujet qui parle et qui incorporent la violence et la jouissance de l'événement d'une parole à une histoire de la vérité.

la figure d'Edipe pour Foucault, nous renvoyons à SForzini (A.), Les Scènes de la vérité. Michel Foucault et le théâtre, Lormont, Le Bord de l'eau, « Diagnostics », chapitre 3.

${ }^{45}$ Sept propos..., p. 43.

${ }^{46}$ Leçons sur la volonté de savoir, op. cit., Leçon du 13 janvier 1971, p. 59.

${ }^{47}$ Ibid., p. 61. 